\title{
Bedside Ultrasonographic Evaluation of Skull Fractures in Pediatric Head Trauma Patients Admitted to Emergency Department
}

\author{
Emre Gökçen ${ }^{1}$, Atakan Savrun ${ }^{2}$ \\ ${ }^{1}$ Department of Emergency Medicine Address: Department of Emergency Medicine, Bozok University Faculty of Medicine, \\ Yozgat, Turkey. \\ ${ }^{2}$ Department of Emergency MedicineAddress: Department of Emergency Medicine, Ordu University Faculty of Medicine, Ordu, \\ Turkey.
}

Received: 15 November 2019, Accepted: 09 December 2019, Published online: 31 December 2019

(C) Ordu University Institute of Health Sciences, Turkey, 2019

\begin{abstract}
Objective: Among children with minor head trauma, the incidence of skull fractures is increased especially in those under one year of age. Several studies investigated the association between skull fracture and traumatic brain injury. In the present study, we aimed to test the potential of ultrasonography in detecting linear skull fracture in pediatric patients with minor head trauma.

Methods: Patients under the age of 18 years with minor head trauma who presented to the emergency units of Bozok University Faculty of Medicine or Ordu University Training and Research Hospital between March 1, 2019 and November 1, 2019 and were planned to undergo head CT by their responsible physicians were included in the present study. The exclusion criteria were a Glasgow Coma Scale (GCS) score $<14$, traumatic open skull deformity, depressed skull fracture, penetrating head trauma, neurological deficits, and hemodynamic instability.

Results: A total of 62 patients (\%64,5 males) with a mean age of 7.29 years were included. The sensitivity and specificity of ultrasound in detection of fractures were \%84.6 (\% 95 CI: 65.13- 95.6) and \% 94.4 (\% 95 CI: 81.3-99.3), respectively.

Conclusion: In conclusion, we suggest that the use of ultrasound before CT examination in patients with minor head trauma may be a helpful means to detect cranial fractures.
\end{abstract}

Key words: Ultrasonographic, Pediatric Head Trauma Patients, Emergency Department

Suggested Citation: Gokcen E, SavrunA. Bedside Ultrasonographic Evaluation of Skull Fractures in Pediatric Head Trauma Patients Admitted to Emergency Department. Middle Black Sea Journal of Health Science, 2019; 5(3):233-238.

\section{Address for correspondence/reprints:}

Emre Gökçen

Telephone number: +90 (505) 5718688

E-mail: emregokcenacl@gmail.com

DOI: $10.19127 / \mathrm{mbsjohs} .647426$

\section{Introduction}

Pediatric head trauma remains to be an important public health problem as a common cause of mortality and morbidity in childhood (Verma et al., 2009; Yanagava and Sakamoto, 2009). Many children are admitted to emergency units with serious traumatic brain injury due to head trauma. The gold standard method to determine the need for surgical treatment is head computed tomography (CT). In addition, CT has an important role in demonstrating the extent of skull fractures and intracranial injury (Fundarò et al., 2012). Whereas the indications for $\mathrm{CT}$ are established in moderate 
and severe traumatic brain injuries, there is no widely accepted assessment tool in the literature about the use of CT in minor head trauma (Siaarti, 2004; Klemetti et al., 2009; Sömez et al., 2018). In this context, physicians in emergency units may have difficulty deciding whether or not to perform head CT in patients with minor head trauma. Among the underlying causes of this difficulty are the inability of a routine physical examination in reflecting brain injury and the risk of radiation exposure, need for sedation, and increased cost associated with CT (Stein et al., 1991; Stein, 1992).

Among children with minor head trauma, the incidence of skull fractures is increased especially in those under one year of age (Greenes and Schutzman, 1997). Skull fractures can be classified as linear, depressed, and basilar and $75 \%$ of all skull fractures are linear in nature (Atabaki, 2007). Several studies investigated the association between skull fracture and traumatic brain injury (Greenes and Schutzman, 1991; Schutzman and Greenes, 2001; Boran et al., 2006). In addition, coexistence of a scalp hematoma with childhood head trauma is a possible indicator of skull fracture (Schutzman and Greenes, 2001; Greenes and Schutzman, 2001). From this point on, the detection of skull fractures in pediatric minor head trauma patients with scalp hematoma using ultrasonography may facilitate risk estimation and aid in the decision to perform further imaging (CT). In the present study, we aimed to test the potential of ultrasonography in detecting linear skull fracture in pediatric patients with minor head trauma.

\section{Methods}

This prospective study was started after Bozok University Ethics Committee approved the study with the approval code "2017-kaek189_2019.02.28_24". Patients under the age of 18 years with minor head trauma who presented to the emergency units of Bozok University Faculty of Medicine or Ordu University Training and Research Hospital between March 1, 2019 and November 1, 2019 and were planned to undergo head CT by their responsible physicians were included in the present study. Other inclusion criterion was the presence of scalp hematoma, soft tissue swelling, or localized tenderness on the head because it is not feasible to screen the whole head for fractures by ultrasonography. The exclusion criteria were a Glasgow Coma Scale (GCS) score <14, traumatic open skull deformity, depressed skull fracture, penetrating head trauma, neurological deficits, and hemodynamic instability.
The HM70A with Plus ultrasound system (Samsung Medison Co., Ltd., Seoul, Korea) was used for ultrasonography and a 7-12 MHz linear probe was used to scan the skull for fractures. The ultrasonography examination was performed beginning on the areas with scalp hematomas, abrasions, and where focal tenderness was present. In case of scalp hematomas, the skull was examined in a perpendicularly oriented fashion on transverse and sagittal-coronal planes between the boundaries of the scalp hematoma. If the area to be examined is over a cranial suture, confirmation was performed from the contralateral area. Fracture lines, bone cortex irregularities, step-off sign, and free bone fragments were recorded. The location of fracture on ultrasound examination was compared with head CT images. Results of the ultrasound examination did not change the patients' diagnosis or treatment algorithm.

\section{Statistical analysis}

The categorical data were presented with numbers and percentages. We calculated the ultrasonographic performance characteristics including sensitivity, specificity, accuracy, positive and negative predictive values with $95 \%$ confidence intervals. The IBM SPSS 26 program (SPSS Inc., Chicago, IL) was used for statistical analyses.

\section{Results}

A total of 62 patients (64.5\% males) with a mean age of 7.29 years were included. The distribution of patients with respect to age groups is shown in Table 1. The frontal bone was the most frequent site of trauma followed by the parietal, occipital, and temporal bones, respectively.

Table 1. Baseline characteristics

\begin{tabular}{cc}
\hline Study Group & $\mathbf{n = 6 2}$ \\
\hline Sex, $\mathbf{n}(\boldsymbol{\%})$ & \\
Male & $40(64,5)$ \\
Female & $22(35,5)$ \\
Age, $\mathbf{n}(\boldsymbol{\%})$ & \\
$0<2$ & $11(17,7)$ \\
$2<5$ & $14(22,6)$ \\
$5<10$ & $21(33,9)$ \\
$10<15$ & $12(19,4)$ \\
$15<18$ & $4(6,5)$ \\
Injury site, $\mathbf{n}(\boldsymbol{\%})$ & \\
Temporal & $10(16,1)$ \\
Occipital & $11(17,7)$ \\
Parietal & $16(25,8)$ \\
Frontal & $25(40,3)$ \\
\hline
\end{tabular}


On the CT examinations 26 of the patients (42\%) were found to have fractures and the remaining 36 $(58 \%)$ did not have fractures. Ultrasound examination achieved to detect fractures in 22 of these 26 patients and failed to show the fractures in the remaining four patients (Table 2) (figure 1). On the other hand, two out of 36 patients without a fracture on CT examination were reported to fractures on ultrasound examination. The sensitivity and specificity of ultrasound in detection of fractures were $84.6 \%$ (95\% CI: 65.13- 95.6) and \% 94.4\% (95\% CI: 81.3-99.3), respectively. The positive and negative predictive values and accuracy for ultrasound examination in detection of fractures were $91.7 \%$ (95\% CI: 74-98), 89.4\% (95\% CI: 78-96), and 90.3\% (95\% CI 80.1-96.4), respectively.

Table 2. Comparison of ultrasound with tomography to determine skull fracture

\begin{tabular}{lcc}
\hline & CT (+) & CT (-) \\
\hline USG (+) & 22 & 2 \\
\hline USG (-) & 4 & 34 \\
\hline Sensitivity, \% (\%95 CI) & $84.6(65.1-95.6)$ \\
\hline $\begin{array}{l}\text { Specificity, \% (\%95 CI) } \\
\text { (\%95 CI) }\end{array}$ & $94.4(81.3-99.3)$ \\
\hline $\begin{array}{l}\text { Negative predictive value, \% } \\
\text { (\%95 CI) }\end{array}$ & $91.7(73.9-97.7)$ \\
\hline $\begin{array}{l}\text { Accuracy, \% (\%95 CI) } \\
\text { p-Value* }\end{array}$ & $90.2(80.1-96.4)$ \\
\hline
\end{tabular}

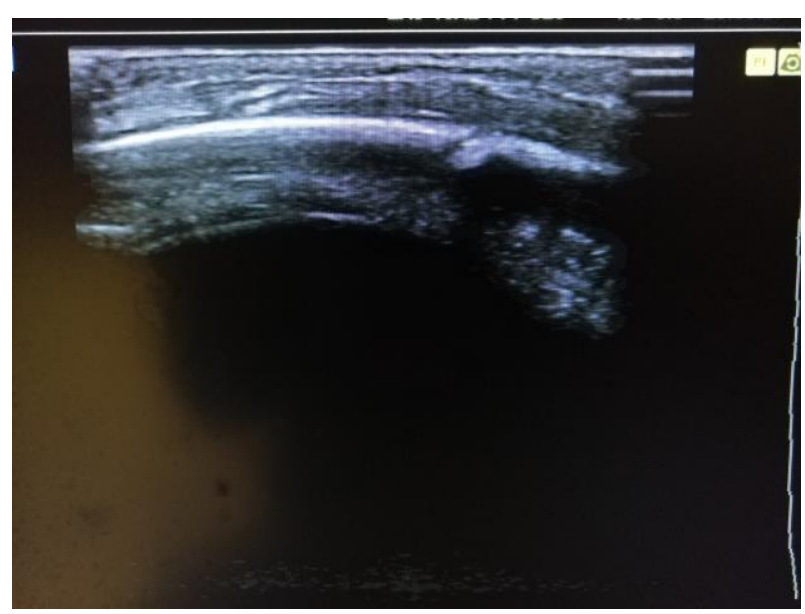

Figure 1. Ultrasonographic image of a six-year-old male patient with frontal skull fracture

\section{Discussion}

In the present study, we primarily demonstrated that ultrasonography is an effective tool to detect skull fractures in childhood minor head trauma. Secondly, we found that the specificity of ultrasound was higher than its sensitivity in detecting skull fractures, hence ultrasound seems to be more effective in excluding fractures in patients with minor head trauma. However, the low prevalence of fractures in the present study may also be the cause of this high specificity. It has been previously reported that specificity increases in case of a low prevalence (Brenner and Gefeller, 1997). Therefore, the prevalence rates should be taken into consideration when using ultrasound to detect/exclude cranial fractures.

In the literature, the number of studies regarding the detection of skull fractures using ultrasound are low. In one study, 69 patients under the age of 21 years were included and eight of these patients $(12 \%)$ were found to have fractures (Rabiner et al., 2013). The sensitivity and specificity of ultrasound in detecting fractures were $88 \%$ (95\% CI 53-98) and 97\% (9\%5 CI: 89-99), respectively. In another study, 55 patients under the age of 18 years underwent bedside ultrasound examination and 35 of these patients $(63.6 \%)$ were found to have fractures (Parri et al., 2013). The sensitivity and specificity of ultrasound in detecting fractures were $100 \%$ (95\% CI 88.2-100) and 95\% (9\%5 CI: 7599.9), respectively. Another study in 46 subjects under the age of 18 years, 11 of the study population (24\%) were found to have fractures (Riera and Chen, 2012). In that study, the sensitivity and specificity of ultrasound in detecting cranial fractures were $82 \%$ (95\% CI: 48- 98) and 94\% (95\% CI: 79-99), respectively. In the present study, the sensitivity and specificity of ultrasound in terms of cranial fracture detection was comparable with the findings of the aforementioned studies. However, ultrasound is an operator dependent modality and there is need for large scale studies to reach a conclusion regarding the exact role of ultrasound in cranial fracture detection.

Ultrasound has many advantages over CT such as the absence of radiation risk, repeatability, and cost-effectiveness (Weinberg, 2010). Sedation of the patient may be required for CT imaging in the pediatric population. Sedation of young children may increase the risk of complications and may cause difficulty in surveillance by affecting the state of consciousness (Sanborn, 2005). On the other hand, ultrasound examination does not require sedation and may facilitate clinical follow up of 
these children by emergency unit physicians. Another advantage of using ultrasound is that it may be performed at bedside and obviates the need to move the patient from the emergency unit (Coskun, 2018). We think that these advantages may prove helpful in early and accurate decision making by emergency unit physicians.

The primary task of the emergency physician in head trauma is to determine the presence of lifethreatening problems. Current clinical and physical examination findings of the patients may be inadequate in the management of patients with minor head trauma. In the recent years, the rates of unnecessary head CT examination in head trauma patients is rather high and most of these examinations do not reveal pathological findings that necessitate surgical intervention (Blackwell et al., 2007; Atmis et al., 2016; Zulfiqar et al.,2017). In a study, only $6 \%$ of patients with minor head trauma were found to have pathological findings on CT (Mannix et al., 2012). In another study, the authors stated that approximately $69 \%$ of patients with minor head trauma did not require CT examination (Fundaro et al., 2012). In another study, $90 \%$ of head CT examinations were reported as normal in patients with minor head trauma (Atabaki, 2007). Performance of CT scans in children with minor head trauma should be reduced to protect children from the hazardous effects of radiation. However, widely accepted management algorithms are not available at the present (Er et al., 2013). We suggest that performance of ultrasound in minor head trauma patients may obviate the need for a CT examination when no fracture is detected on ultrasound. In addition, the detection of a fracture on ultrasound examination may prompt the physician to perform urgent further imaging.

On some occasions a CT device may not be available in the hospital and conventional radiography may be preferred in these centers. However, several studies indicate that the specificity and sensitivity of conventional radiography is low in minor head trauma patients (Feuerman et al., 1988; Thiruppathy and Muthukumar, 2004). The management of a patient with minor head trauma in a center without a CT device is controversial and it is hard to determine which patient to discharge home because of low risk and which patient to refer for further examination and treatment. However, although ultrasound examination may aid in the management of patients with head trauma by detecting or excluding fracture, its exact role is not clear. We found a relatively high sensitivity and specificity for ultrasound in patients with minor head trauma, but further larger scale studies are needed to reach a strict conclusion in this topic.

The most important limitation for ultrasound to detect cranial fractures in patients with scalp hematoma is the fact that the fracture line may not be under the scalp hematoma and rather near the boundary of it (Arnholz et al., 1998). Another limitation is when a tiny fracture connects with a cranial suture (Furuya et al., 1984). In the present study, the fracture line was near the boundary of scalp hematoma on $\mathrm{CT}$ in a patient in whom ultrasound failed to detect the fracture. Another two patients in whom ultrasound failed to detect the fracture had linear nondisplaced thin fractures. A sound knowledge of cranial suture anatomy may avoid this type of confusion. As a matter of fact, in the present study, the ultrasound operator evaluated the suture line of these two patients as fractures. Patient cooperation is also important for an effective screening. The ultrasound operator should carefully scan the area in all directions. In the present study, one patient in whom the fracture could not be detected by ultrasound examination had a poor cooperation. Another difficulty in ultrasound scanning is the application of ultrasound gel on the hair. Fluid-filled gloves or special pads may be used to overcome this problem. However, when mirrorimage artefact should be kept in min when using a fluid-filled device on a scalp hematoma.

Conclusions

In conclusion, we suggest that the use of ultrasound before CT examination in patients with minor head trauma may be a helpful means to detect cranial fractures. We also believe that many unnecessary CT examinations may be avoided in patients with minor head trauma if an appropriate scoring system is established. We also believe that ultrasound examination would play a key role in the risk calculation and management in patients with minor head trauma provided that its exact role in this condition is established with further studies. We believe that our work would shed light on future studies in this topic. 
Ethics Committee Approval: Ethics committee approval was received for this study from Yozgat Clinical Research Ethics Committee of Bozok University (Ethichs No: 2019-01-07).

Peer-review: Externally peer-reviewed.

Author Contributions: Externally peer-reviewed. Author Contributions: Concept - GE, AS Design GE, AS; Supervision GE, AS; Materials- GE, AS; Data Collection and/or Processing- GE, AS; Analysis and/or Interpretation- GE, AS; Literature Review- GE; Writing- GE, AS; Critical Review GE, AS.

Conflict of Interest: No conflict of interest was declared by the authors.

Financial Disclosure: The authors declared that this study hasn't received no financial support.

\section{References}

Arnholz D, Hymel KP, Hay TC, Jenny C. Bilateral pediatric skull fractures: accident or abuse? Journal of Trauma and Acute Care Surgery. 1998;45(1):172-4.

Atabaki SM. Pediatric head injury. Pediatrics in Review. 2007;28(6):215.

Atmis A, Tolunay O, Çelik $\mathrm{T}$, Gezercan $\mathrm{Y}$, Dönmezer Ç, Resitoglu S, et al. Dilemma in Pediatric Head Trauma: Is Cranial Computed Tomography Necessary or not in Minor Head Traumas? Child Emergency and Intensive Care.2016;3(1):27.

Blackwell CD, Gorelick M, Holmes JF, Bandyopadhyay S, Kuppermann N. Pediatric head trauma: changes in use of computed tomography in emergency departments in the United States over time. Annals of emergency medicine. 2007;49(3):320-4.

Boran BO, Boran P, Barut N, Akgun C, Celikoglu $\mathrm{E}$, Bozbuga M. Evaluation of mild head injury in a pediatric population. Pediatric neurosurgery. 2006;42(4):203-7.

Brenner H, Gefeller O. Variation of sensitivity, specificity, likelihood ratios and predictive values with disease prevalence. Statistics in medicine. 1997;16(9):981-91.

Coskun F. Emergency Ultrasonography (PoCUS) in the Emergency Medicine Training Curriculum (PoCUS). Turkey Clinics Emergency MedicineSpecial Topics. 2018;4(1):106-12.

Er A, Akman C, Alataș I, Unlu HB, Ceylan I, Gün $\mathrm{C}$, et al. Should Children with Minor Head Injury Routinely Have CT Scan? JOPP. 2013;5(3):1315 .
Feuerman T, Wackym PA, Gade GF, Becker DP. Value of skull radiography, head computed tomographic scanning, and admission for observation in cases of minor head injury. Neurosurgery. 1988;22(3):449-53.

Fundaro C, Caldarelli M, Monaco S, Cota F, Giorgio V, Filoni S, et al. Brain CT scan for pediatric minor accidental head injury. An Italian experience and review of literature. Child's Nervous System. 2012;28(7):1063-8.

Fundarò $\mathrm{C}$, Caldarelli $\mathrm{M}$, Monaco $\mathrm{S}$, Cota $\mathrm{F}$, Giorgio V, Filoni S, et al. Brain CT scan for pediatric minor accidental head injury. An Italian experience and review of literature. Child's Nervous System. 2012;28(7):1063-8.

Furuya Y, Edwards MS, Alpers CE, Tress BM, Ousterhout DK, Norman D. Computerized tomography of cranial sutures: Part 1: Comparison of suture anatomy in children and adults. Journal of neurosurgery. 1984;61(1):538.

Greenes DS, Schutzman SA. Clinical indicators of intracranial injury in head-injured infants. Pediatrics. 1999;104(4):861-7.

Greenes DS, Schutzman SA. Clinical significance of scalp abnormalities in asymptomatic headinjured infants. Pediatric emergency care. 2001;17(2):88-92.

Greenes DS, Schutzman SA. Infants with isolated skull fracture: what are their clinical characteristics, and do they require hospitalization? Annals of emergency medicine. 1997;30(3):253-9.

Klemetti S, Uhari M, Pokka T, Rantala $H$. Evaluation of decision rules for identifying serious consequences of traumatic head injuries in pediatric patients. Pediatric emergency care. 2009;25(12):811-5.

Mannix R, Meehan WP, Monuteaux MC, Bachur RG. Computed tomography for minor head injury: variation and trends in major United States pediatric emergency departments. The Journal of pediatrics. 2012;160(1):136-9. e1.

Parri N, Crosby BJ, Glass C, Mannelli F, Sforzi I, Schiavone R, et al. Ability of emergency ultrasonography to detect pediatric skull fractures: a prospective, observational study. The Journal of emergency medicine. 2013;44(1):135-41.

Rabiner JE, Friedman LM, Khine H, Avner JR, Tsung JW. Accuracy of point-of-care ultrasound for diagnosis of skull fractures in children. Pediatrics. 2013;131(6):e1757-e64. 
Riera A, Chen L. Ultrasound evaluation of skull fractures in children: a feasibility study. Pediatr Emerg Care. 2012;28(5):420-5.

Sanborn PA, Michna E, Zurakowski D, Burrows PE, Fontaine PJ, Connor L, et al. Adverse cardiovascular and respiratory events during sedation of pediatric patients for imaging examinations. Radiology. 2005;237(1):288-94.

Schutzman SA, Greenes DS. Pediatric minor head trauma. Annals of emergency medicine. 2001;37(1):65-74.

Siaarti Lg. Siaarti-Sarnepi guidelines for the management of severe pediatric head injury. Minerva anestesiologica. 2004;70:549-604.

Sönmez FT, Kılıçaslan Ö, Yalçın G. 2 The Role of Computed Tomography in the Evaluation of Head Trauma in Children Under Age. Konuralp Medical Journal. 2018;10(3):294-7.

Stein SC, O'Malley KF, Ross SE. Is routine computed tomography scanning too expensive for mild head injury? Annals of emergency medicine. 1991;20(12):1286-9.

Stein SC, Ross SE. Mild head injury: a plea for routine early CT scanning. The Journal of trauma. 1992;33(1):11-3.

Thiruppathy S, Muthukumar N. Mild head injury: revisited. Acta neurochirurgica. 2004;146(10):1075-83.

Verma S, Lal N, Lodha R, Murmu L. Childhood trauma profile at a tertiary care hospital in India. Indian pediatrics. 2009;46(2).

Weinberg ER, Tunik MG, Tsung JW. Accuracy of clinician-performed point-of-care ultrasound for the diagnosis of fractures in children and young adults. Injury. 2010;41(8):862-8.

Yanagawa Y, Sakamoto T. Characteristics of pediatric trauma in an urban city in Japan. Pediatric emergency care. 2009;25(9):572-4.

Zulfiqar M, Kim S, Lai JP, Zhou Y. The role of computed tomography in following up pediatric skull fractures. American journal of surgery. 2017;214(3):483-8. . 